rheuma plus $2021 \cdot 20: 135-137$

https://doi.org/10.1007/s12688-021-00457-4

(c) Springer-Verlag GmbH Austria, ein Teil von Springer Nature 2021

Liebe Leserinnen und Leser,

Sommer 2021 - ein Sommer wie damals? Nun ja, die Corona-Zahlen sinken glücklicherweise - jahreszeitlich bedingt. Gott sei Dank sind wir im griechischen Alphabet auch erst bei Delta angekommen, in Anbetracht der Vielzahl von Virusmutationen, die wir in den nächsten Jahren noch zu erwarten haben. Aber was ist normal? Ist es normal, dass wir täglich Erkrankungsmeldungen von einer einzigen Erkrankung veröffentlicht bekommen? Warum werden nicht die Anzahl der täglichen Cholecystektomien, der Herzkatheter und Stentimplantationen pro Tag, ja die Anzahl der Neudiagnosen rheumatoide Arthritis veröffentlicht? Sind alle anderen Erkrankungen zweite Wahl? Vielleicht könnte man ja die altehrwürdige Wiener Zeitung wieder als Organ zur Veröffentlichung des täglichen Krankheitsberichtes in Österreich verwenden, anstatt sie einzustellen? Man wird sich entscheiden müssen: Normalität mit COVID-19 oder Abnormalität wegen COVID-19. Ich jedenfalls bin überzeugt, dass die Menschheit reif ist für die Realität und ihr Schicksal selbst bestimmen kann, also nicht von einigen Wenigen besonders „beschützt“ werden muss.

\section{Das Editorial Board wird erweitert}

Doch jetzt genug von Corona - bei rheuma plus verändert sich mit dieser Ausgabe Wesentliches. Nach fast zehn Jahren alleiniger Herausgeberschaft bin ich froh und auch ein bisschen stolz bekanntgeben zu können, dass sich das Editorial Board um drei illustre Persönlichkeiten aus der österreichischen

\title{
Burkhard Leeb
}

Institut für Klinische Rheumatologie, Karl Landsteiner Gesellschaft, Hollabrunn, Österreich

\section{Die Zeit eilt im Sauseschritt - rheuma plus eilt mit}

Rheumatologie erweitern wird. Dies soll auch der Vielgestaltigkeit der Rheumatologie und den unterschiedlichen Institutionen, an denen Rheumatologie betrieben wird, Rechnung tragen. Stolz macht es mich, dass alle drei Herren ohne Zögern prompt zugesagt haben bei rheuma plus mitzuarbeiten.

Christian Huemer (• Abb. 1) ist an der MedUni Wien ausgebildeter Kinderarzt. An der kanadischen University of British Columbia hat er seine Ausbildung in Richtung Pädiatrische Rheumatologie erweitert und auch Forschungen auf diesem Gebiet begonnen. Er habilitierte 2003 für das Fach Kinder- und Jugendheilkunde. Seit mittlerweile knapp 20 Jahren leitet Christian Huemer die Abteilung für Kinder- und Jugendheilkunde in Bregenz und ist leitender Arzt für Pädiatrische Rheumatologie am Ostschweizer Kinderspital St. Gallen. Ich freue mich, dass Christian Huemer die Einladung in das rheuma plus-Board angenommen hat, wo er zukünftig der Pädiatrischen Rheumatologie den ihr zustehenden Raum geben wird.

Raimund Lunzer (• Abb. 2) ist Arzt für Allgemeinmedizin und Facharzt für Innere Medizin sowie Rheumatologie. Er ist unter anderem Berater des österreichischen Morbus Bechterew-Vereins und der Österreichischen Rheumaliga. Er unterrichtet an der Fachhochschule Joanneum/Steiermark die Fachrichtungen Ergotherapie und Diätologie. Seit 2008 ist Dr. Lunzer Oberarzt bei den Barmherzigen Brüdern in Graz/Leitung Rheumatologie. Zu seinen Forschungs-Schwerpunkten zählen die Psoriasis-Arthritis bzw. Gicht. Seine Aufgabe wird es sein, die Belange der krankenhausbasierten Rheumatologie zu repräsentieren.
Rudolf Puchner (•Abb. 3) ist seit mehr als 20 Jahren niedergelassener Internist und Rheumatologe in Wels. Er war von 2009 bis 2020 im Vorstand der österreichischen Gesellschaft für Rheumatologie und Rehabilitation (ÖGR) und von 2016 bis 2018 deren Präsident. 2020 erfolgte die Habilitation für das Fach Rheumatologie. Einer seiner Forschungsschwerpunkte ist die rheumatologische Versorgungsforschung. Ein besonderes Anliegen ist Dr. Puchner, junge Mediziner für das Fach Rheumatologie zu begeistern und zu gewinnen. Aus diesem Grund wurde während seiner Präsidentschaft die Rheuma Summer School für Medizinstudenten in höheren Semestern gegründet. Ein großer Teil seiner Arbeit liegt naturgemäß in der Betreuung und Behandlung von Patienten mit entzündlich rheumatischen Erkrankungen - er hat daher die Einladung als Board Mitglied zu wirken gerne angenommen um einen Beitrag zu leisten, die Kenntnisse über rheumatische Erkrankungen auch interdisziplinär zu erweitern.

Ja, und ich bin froh nach Jahren als Einzelkämpfer so profunde Unterstützung dabei zu bekommen, rheuma plus weiterzuentwickeln.

Der Bogen dieser Ausgabe spannt sich von der Genetik, die bei Erkrankungen des Knochens und des Bindegewebes oft eine wesentliche und manchmal auch ursächliche Rolle spielt, bis hin zur Beobachtung von Schüben nach Impfungen bei Patientinnen und Patienten mit Autoimmunerkrankungen. Katharina Rötzer und Gökhan Uyanik, Wien, geben in ihrem Beitrag einen Überblick über den aktuellen Stand der genetischen Analysen in der Osteologie mit dem Schwerpunkt aufklinisch relevante und aus genetischer 


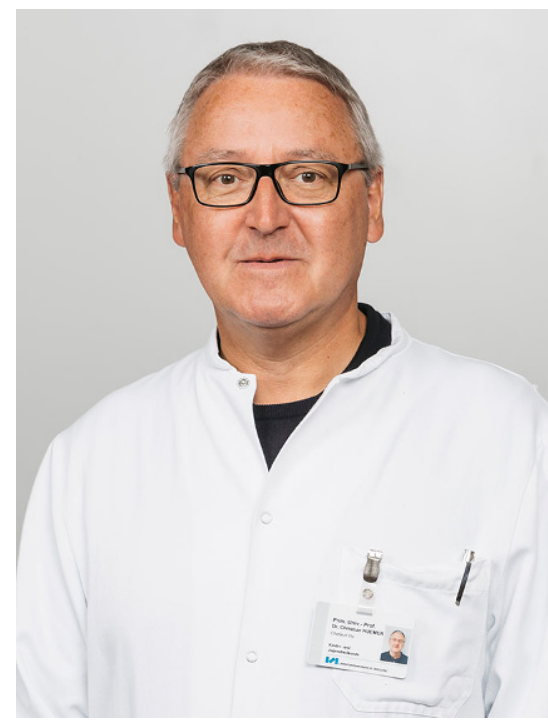

Abb. 1 ॥ Prim. Prof. Dr. Christian Huemer. (c) Lisa Mathis Fotografie

Sicht gut beurteilbare Fragestellungen. Die Maßnahmen aufgrund der Coronapandemie haben uns sehr eindringlich vor Augen geführt, dass alternative Betreuungsformen, wie z.B. EDV-gestütztes Patientenmonitoring, plötzlich von schwer vorstellbar zu alltagstauglich mutierten.

Johannes Pallua und Michael Schirmer, Innsbruck, zeigen in ihrem Beitrag über die Rolle der Dokumentations-IT in der Rheumatologie die Möglichkeiten, aber auch die Probleme und Schwächen, dieser Technologie auf. Jedenfalls ist anzunehmen, dass die elektronische Datenerfassung und Dokumentation zu einem integralen Bestandteil zukünftiger Betreuungsformen werden wird.

Auch um Patientenbetreuung und um die Anwendung von Behandlungsempfehlungen im klinischen Alltag dreht sich der Beitrag von Andreas Kerschbaumer und Daniel Aletaha, Wien. Es freut mich besonders, dass der jüngst ernannte Ordinarius für Rheumatologie in Wien sich dieses, des wahrscheinlich wichtigsten und vermutlich auch schwierigsten, Themas gemeinsam mit seinem Mitarbeiter annimmt. Der Transfer von mehr oder weniger abstraktem, theoretischem Wissen in die klinische Praxis, die Übertragung auf die Behandlung des einzelnen Patienten mit seinen Wünschen, Erwartungen, Ängsten und Trade-Offs ist wohl das Herausforderndste für den nieder-

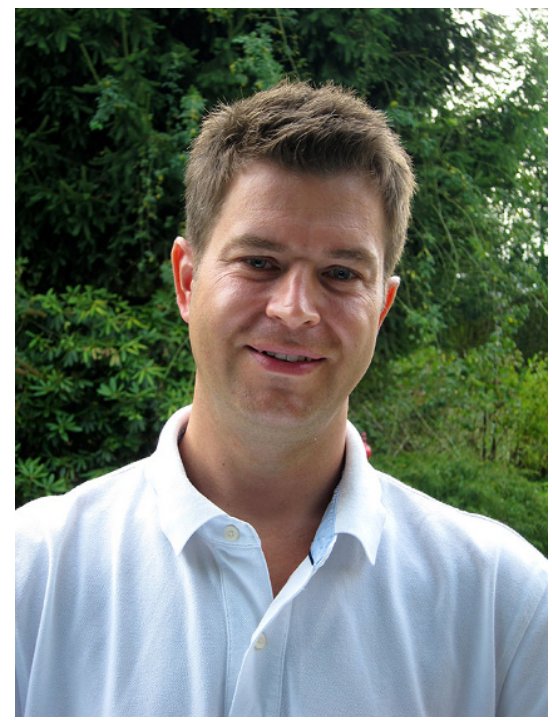

Abb. 2 \ OA Dr. Raimund Lunzer. @ Privat

gelassenen Arzt. In diesen Kontext passt auch der Brief an den Herausgeber, den Raimund Lunzer, noch vor der Einladung in das Editorial Board, über schubhafte Verschlechterungen rheumatischer Erkrankungen nach Impfungen verfasst hat. In dieser Ausgabe in gedruckter Form veröffentlicht, gibt der Brief vielleicht Anstoß zu weiterer Diskussion.

Auch mit dieser Ausgabe hoffen wir wieder Ihr Interesse zu wecken und dem Ziel von rheuma plus nahe zu kommen, eine fruchtbringende, offene und redliche Diskussion in Gang zu bringen, aus der wir alle Nutzen ziehen können. Wie immer an dieser Stelle möchten wir Sie herzlich dazu einladen, uns Ihre Meinung zu den Beiträgen dieser Ausgabe, wie auch $\mathrm{zu}$ allen rheumatologischen Themen, die Sie für relevant oder für diskussionswert halten, zu schicken. Das ist erfreulicherweise in den letzten Wochen mehrmals geschehen! Die entsprechenden Manuskripte werden zunächst Online First und dann, in einer der nächsten Ausgaben von rheuma plus, in gedruckter Form erscheinen. Sie können in jedem Fall auf einen fairen Review mit entsprechendem Feedback vertrauen. Für Kritik, Hinweise, Zustimmung, wie auch für jeden anderen Beitrag, bedanken wir uns schon im Voraus.

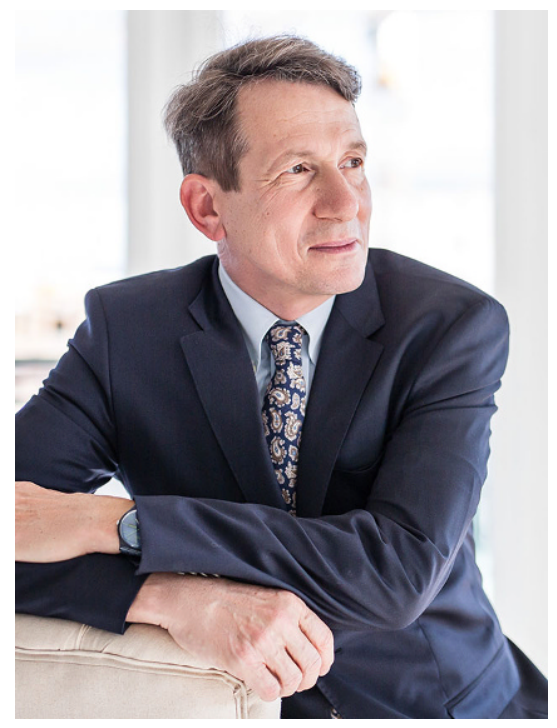

Abb. 3 ム Priv.-Doz. Dr. Rudolf Puchner MSc MBA. (c) Sandra Gehmair

Herzlichst, Ihr

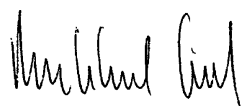

Burkhard Leeb

\section{Korrespondenzadresse}

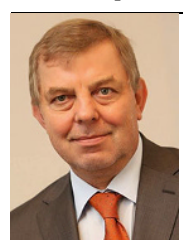

PD Dr. Burkhard Leeb

Institut für Klinische

Rheumatologie, Kar

Landsteiner Gesellschaft

Babogasse 20, 2020 Holla-

brunn, Österreich

() Privat

leeb.rheuma@aon.at

Interessenkonflikt. B. Leeb gibt an, dass kein Interessenkonflikt besteht.

Hinweis des Verlags. Der Verlag bleibt in Hinblick auf geografische Zuordnungen und Gebietsbezeichnungen in veröffentlichten Karten und Institutsadressen neutral. 
Hier steht eine Anzeige.

\section{曾 Springer}

